\title{
Hirschsprung Disease in an Infant with L1 Syndrome: Report of a New Case and a Novel L1CAM Variant
}

\author{
Teresa Andreone ${ }^{1}$ \\ ${ }^{1}$ Saint Louis University Care - The Physicians of Saint Louis University
}

October 27, 2020

\begin{abstract}
L1 syndrome is an X-linked disorder manifesting with congenital hydrocephalus, adducted thumbs and spasticity. There are rare cases of L1 syndrome and coincident Hirschsprung disease, with mutations in the L1CAM gene thought to underlie both. We present a novel pathogenic L1CAM variant in someone with L1 syndrome and Hirschsprung disease.
\end{abstract}

\section{Introduction}

The L1CAM gene encodes the membrane glycoprotein L1CAM, a calcium-independent cellular adhesion molecule involved in neuronal development. The L1CAM cell adhesion molecule is found on the X chromosome in humans (and other mammals) and has a 1253 amino acid protein sequence. The extracellular portion is comprised of six immunoglobulin domains followed by five fibronectin type III domains which are connected to a small intracellular domain by a transmembrane helix (Figure 1). Mutations in the L1CAM gene cause L1 syndrome, which encompasses a spectrum of disease that includes four major X-linked conditions: X-linked congenital hydrocephalus due to stenosis of the aqueduct of Sylvius (HSAS; OMIM \#307000); mental retardation, aphasia, shuffling gait and adducted thumbs syndrome (MASA; OMIM \#303350); X-linked complicated hereditary spastic paraplegia type 1 (SPG1; OMIM\#303350); and X-linked complicated agenesis of the corpus callosum (OMIM \#304100) ${ }^{1}$. More than 220 disease-causing variants in the L1CAM gene have been identified as causing the four major phenotypes of L1 syndrome ${ }^{2}$. Additionally, there are reports suggesting that additional mutations in $L 1 C A M$ can cause mild behavioral and intellectual impairment ${ }^{3}$. The phenotypic manifestation of L1 syndrome is highly variable, even among patients who share the same pathogenic variant.

Hirschsprung disease (HD) is a congenital malformation of the enteric nervous system that has a prevalence of approximately 1 in 5000 live births in the United States ${ }^{4}$. Failure of neural crest cells to migrate the entire length of the developing gastrointestinal tract results in a segment of distal colon that lacks ganglion cells in the submucosal and myenteric plexuses, thus creating a functional obstruction. There are at least 12 genes in which known pathogenic mutations have been causatively associated with $\mathrm{HD}^{5}$. The incomplete penetrance and variable presentation of the disease, however, suggests a non Mendelian, model of inheritance.

Hirschsprung disease has previously been associated with L1 syndrome where children have been identified who had both verified $L 1 C A M$ mutations and biopsy-proven $\mathrm{HD}^{1,2,6-10}$. Studies have suggested that L1CAM acts as a modifier gene for several HD-causative genetic mutations, such as RET, SOX10 and several genes that encode for proteins in the endothelin signaling pathway ${ }^{5,11}$. Evidence from a murine model suggests that interference of L1 protein function during embryogenesis impairs neural crest cell migration in the developing gut ${ }^{12}$. Resected sigmoid colon from infants with HD has been shown to lack expression of L1 protein via immunohistochemical staining ${ }^{13}$. Nevertheless, in humans a pathogenic variant in L1CAM has 
not been identified whose causative presence always leads to HD. Therefore, a biopsy is always required to diagnose HD, even in patients with genetically diagnosed L1 syndrome.

Here we present a rare case of an infant with clinically diagnosed L1 syndrome who was found to have biopsy-proven Hirschsprung disease during admission for a ventriculoperitoneal shunt infection. Subsequent genetic confirmation of L1 syndrome by targeted sequencing of the $L 1 C A M$ gene revealed a novel pathogenic variant (c.934T >C; p.Cys312Arg). We discuss the possible role of pathogenic L1CAMmutations in the pathogenesis of HD. We also emphasize that clinicians should have a high index of suspicion for HD when caring for patients with any form of L1 syndrome.

\section{Case Report}

The proband, who was the first child born to non-consanguineous Caucasian parents, was diagnosed with hydrocephalus in utero. On DOL 1, magnetic resonance imaging of the brain demonstrated profound ventriculomegaly and the corpus callosum was not able to be visualized (Figure 2A). A ventriculoperitoneal (VP) shunt was placed on DOL 2 to manage hydrocephalus. The child was also diagnosed with bilaterally adducted thumbs (Figure 2B). Notably, the patient's maternal uncle was also born with hydrocephalus and bilaterally adducted thumbs and remains wheelchair-bound with severe intellectual disability. During his fifth week of life, the patient was admitted to hospital with concern for VP shunt malfunction and infection. The VP shunt was removed and an external ventricular drain was placed. He remained in hospital for full treatment of Staphylococcus epidermidis ventriculitis/meningitis. The patient had a prolonged hospital course typified by the need for mechanical ventilation and sedation. The external ventricular drain was removed prior to discharge, followed by a "shunt holiday" until a new VP shunt was placed at 11.5 weeks of age.

During the patient's admission for ventriculitis/meningitis, he developed a distended abdomen and failure to pass stool. Abdominal radiographs demonstrated profoundly dilated loops of colon (Figure 2C). A diagnosis of HD was considered but was initially deemed unlikely given the patient's late presentation. In parental retrospect and on chart review of his NICU stay, it was determined that the patient had suffered from intermittent constipation with abdominal distension during his first month of life. Due to concern for L1 syndrome with coincident HD, a rectal biopsy was ultimately performed at 6.5 weeks of age. The rectal biopsy was significant for the absence of ganglion cells on 76 consecutive sections of rectal biopsy and for the absence of typical neuritic fibers in lamina propria. The calretinin and acetylcholine esterase immunohistochemical staining were both negative and prominent hypertrophic nerve trunks were seen in the muscularis mucosa (Figure 3). These findings were pathognomonic for Hirschsprung disease. Subsequent targeted sequencing of the patient's $L 1 C A M$ gene demonstrated a previously-unreported variant in exon 8 of the L1CAM gene (NM_000425.3): c.934T >C; p.Cys312Arg (C312R).

\section{Discussion}

There are 13 previously described cases of pathogenic L1CAM mutations in patients with both L1 syndrome and Hirschsprung disease (Table 1) ${ }^{1,2}, 6-8,14-19$. Here, we describe a new patient with genetically confirmed L1 syndrome and biopsy-proven HD. We also report a novel missense point mutation (c.934T $>$ C) that results in a newly-described amino acid sequence change (p.Cys312Arg) in exon 8 of the L1CAM gene. This exon encodes for the Immunoglobulin (Ig) 3 domain of the L1CAM protein (Figure 1). Previous studies have linked this domain to homophilic interactions with L1CAM proteins on neighboring cells ${ }^{20}$. Of the previously reported $L 1 C A M$ mutations in patients with L1 syndrome and $\mathrm{HD}$, the majority affect the fibronectin type 3 domain, with only one mutation reported by Basel-Vanagaite et al. 2006 affecting the Ig 3 domain (Table 1) ${ }^{1}$. Notably, the mutation reported by Basel-Vanagaite et al. 2006 was present in two half-siblings, but only one was diagnosed with HD. A different DNA mutation (c.935G $>$ A) and amino acid substitution (p.Cys312Tyr) at the same locus of our patient's mutation was described by Kanemura et al. 2006 , but that case was reported as $\mathrm{L} 1$ syndrome without associated $\mathrm{HD}^{21}$. Thus, mutations that affect the Ig 3 domain of the L1 protein may cause L1 syndrome, but do not necessarily also result in HD. Our patient's $\mathrm{C} 312 \mathrm{R}$ variant results in a non-conservative amino acid substitution of cysteine with arginine, resulting in 
the exchange of an amino acid with an uncharged side chain with one that has a positively charged side chain (Figure 4). This substitution could very likely affect L1CAM protein conformation or disrupt homophilic or heterophilic interactions of L1CAM protein with other proteins, thus altering L1CAM function. Analysis of the c.934T $>$ C point mutation using the Combined Annotation-Dependent Depletion (CADD) tool - an in silicoprediction algorithm that assesses genetic variants as benign or pathogenic - yields a CADD score of 26.6, which corresponds to a likely pathogenic variant ${ }^{22}$. The majority of mutations reported in Table 1 also have CADD scores $>15$, strongly suggesting that L1CAM mutations are indeed pathogenic in each case. Therefore, our patient's novel missense point mutation (c.934T $>$ C) could be considered causative of his L1 syndrome. This mutation might also be implicated in this child's HD.

This case adds to a small number of reports of L1 syndrome with coincident HD, further supporting the hypothesis that $L 1 C A M$ gene mutations may be involved in the pathogenesis of HD, most likely resulting in modified proteins that impact genetically susceptible individuals. Yet, there are hundreds of pathogenic L1CAM mutations described in cases of L1 syndrome without associated $\mathrm{HD}^{1,21,23}$, indicating that the presence of anL1CAM mutation alone is not diagnostic of HD in a child with phenotypic L1 syndrome. We conclude that Hirschsprung disease should be considered and rectal biopsy pursued in all children with intestinal obstructive dysfunction and suspected L1 syndrome. Conversely, diagnosis of L1 syndrome might be considered in children who are diagnosed with HD and exhibit features such as adducted thumbs, frontal bossing or developmental delay. Since L1 syndrome manifests across a wide phenotypic spectrum, cliniciansand pediatricians in particular - should be familiar with the major phenotypes of this disease.

Author Contributions:

T. Gauntner, M. Karumuri, H. Pinz, S. Braddock and T. Andreone identified the case for this case report and contributed to management of the patient. T. Gauntner wrote the case report. T. Andreone and S. Braddock critically reviewed the case report. T. Andreone also revised and edited the case report. T. Andreone, M. Guzman, S. Starnes, S. Besmer provided diagnostic care and assisted with radiology and histopathology images.

Funding Source: No funding was secured for this study.

Financial Disclosure: The authors have no financial relationships relevant to this article to disclose.

Patient consent for publication: Obtained from parents

Conflict of Interest: The authors have no conflicts of interest to disclose.

References

1. Basel-Vanagaite L, Straussberg R, Friez MJ, et al. Expanding the phenotypic spectrum of L1CAMassociated disease. Clinical Genetics . May 2006;69(5):414-419. doi:10.1111/j.1399-0004.2006.00607.x

2. Fernandez RM, Nunez-Torres R, Garcia-Diaz L, Carlos de Agustin J, Antinolo G, Borrego S. Association of X-Linked Hydrocephalus and Hirschsprung Disease: Report of a New Patient With a Mutation in the L1CAM Gene. American Journal of Medical Genetics Part A . Apr 2012;158A(4):816-820. doi:10.1002/ajmg.a.35244

3. Otter M, Wevers M, Pisters M, et al. A novel mutation in L1CAM causes a mild form of L1 syndrome: a case report. Clinical case reports . 2017-Aug 2017;5(8):1213-1217. doi:10.1002/ccr3.1038

4. Amiel J, Sproat-Emison E, Garcia-Barcelo M, et al. Hirschsprung disease, associated syndromes and genetics: a review. Journal of Medical Genetics . Jan 2008;45(1):1-14. doi:10.1136/jmg.2007.053959

5. Wallace AS, Tan MX, Schachner M, Anderson RB. L1cam acts as a modifier gene for members of the endothelin signalling pathway during enteric nervous system development. Neurogastroenterology and Motility . Nov 2011;23(11):E510-E522. doi:10.1111/j.1365-2982.2011.01692.x

6. Okamoto N, Del Maestro R, Valero R, et al. Hydrocephalus and Hirschsprung's disease with a mutation of L1CAM. Journal of Human Genetics . Jun 2004;49(6):334-337. doi:10.1007/s10038-004-0153-4

7. Nakakimura S, Sasaki F, Okada T, et al. Hirschsprung's disease, acrocallosal syndrome, and congenital hydrocephalus: report of 2 patients and literature review. Journal of Pediatric Surgery . May 
2008;43(5)E13. doi:10.1016/j.jpedsurg.2007.12.069

8. Jackson S-R, Guner YS, Woo R, Randolph LM, Ford H, Shin CE. L1CAM mutation in association with X-linked hydrocephalus and Hirschsprung's disease. Pediatric Surgery International . Sep 2009;25(9):823-825. doi:10.1007/s00383-009-2420-0

9. Fernandez RM, Nunez-Torres R, Gonzalez-Meneses A, Antinolo G, Borrego S. Novel association of severe neonatal encephalopathy and Hirschsprung disease in a male with a duplication at the Xq28 region.Bmc Medical Genetics . Sep 22 2010;11137. doi:10.1186/1471-2350-11-137

10. Marin R, Ley-Martos M, Gutierrez G, Rodriguez-Sanchez F, Arroyo D, Mora-Lopez F. Three cases with L1 syndrome and two novel mutations in the L1CAM gene. European Journal of Pediatrics . Nov 2015;174(11):1541-1544. doi:10.1007/s00431-015-2560-2

11. Wallace AS, Schmidt C, Schachner M, Wegner M, Anderson RB. L1cam acts as a modifier gene during enteric nervous system development.Neurobiology of Disease . Dec 2010;40(3):622-633. doi:10.1016/j.nbd.2010.08.006

12. Anderson RB, Turner KN, Nikonenko AG, Hemperly J, Schachner M, Young HM. The cell adhesion molecule L1 is required for chain migration of neural crest cells in the developing mouse gut.Gastroenterology . Apr 2006;130(4):1221-1232. doi:10.1053/j.gastro.2006.01.002

13. Ikawa H, Kawano H, Takeda Y, et al. Impaired expression of neural cell adhesion molecule L1 in the extrinsic nerve fibers in Hirschsprung's disease. Article. Journal of Pediatric Surgery . Apr 1997;32(4):542-545. doi:10.1016/s0022-3468(97)90703-x

14. Okamoto N, Wada Y, Goto M. Hydrocephalus and Hirschsprung's disease in a patient with a mutation of L1CAM. Journal of Medical Genetics . Aug 1997;34(8):670-671. doi:10.1136/jmg.34.8.670

15. Vits L, Chitayat D, Van Camp G, Holden JJA, Fransen E, Willems PJ. Evidence for somatic and germline mosaicism in CRASH syndrome.Human Mutation . 1998 1998;0(SUPPL. 1):S284-S287.

16. Parisi MA, Kapur RP, Neilson I, et al. Hydrocephalus and intestinal aganglionosis: Is L1CAM a modifier gene in Hirschsprung disease? American Journal of Medical Genetics . Feb 15 2002;108(1):5156. doi:10.1002/ajmg.10185

17. Griseri P, Vos Y, Giorda R, et al. Complex pathogenesis of Hirschsprung's disease in a patient with hydrocephalus, vesico-ureteral reflux and a balanced translocation $\mathrm{t}(3 ; 17)(\mathrm{p} 12 ; \mathrm{q} 11)$. European Journal of Human Genetics . Apr 2009;17(4):483-490. doi:10.1038/ejhg.2008.191

18. Takenouchi T, Nakazawa M, Kanemura Y, et al. Hydrocephalus With Hirschsprung Disease: Severe End of X-linked Hydrocephalus Spectrum. Article. American Journal of Medical Genetics Part A . Apr 2012;158A(4):812-815. doi:10.1002/ajmg.a.35245

19. Tegay DH, Lane AH, Roohi J, Hatchwell E. Contiguous gene deletion involving L1CAM and AVPR2 causes X-linked hydrocephalus with nephrogenic diabetes insipidus. American Journal of Medical Genetics Part A . Mar 2007;143A(6):594-598. doi:10.1002/ajmg.a.31536

20. Haspel J, Grumet M. The L1CAM extracellular region: A multi-domain protein with modular and cooperative binding modes. Frontiers in Bioscience-Landmark . Sep 2003;8:S1210-S1225. doi:10.2741/1108

21. Kanemura Y, Okamoto N, Sakamoto H, Shofuda T, Kamiguchi H, Yamasaki M. Molecular mechanisms and neuroimaging criteria for severe L1 syndrome with X-linked hydrocephalus. Journal of Neurosurgery . Nov 2006;105(5):403-412. doi:10.3171/ped.2006.105.5.403

22. Kircher M, Witten DM, Jain P, O'Roak BJ, Cooper GM, Shendure J. A general framework for estimating the relative pathogenicity of human genetic variants. Nature Genetics . Mar 2014;46(3):310-+. doi:10.1038/ng.2892

23. Vos YJ, de Walle HEK, Bos KK, et al. Genotype-phenotype correlations in L1 syndrome: a guide for genetic counselling and mutation analysis. Journal of Medical Genetics . Mar 2010;47(3):169-175. doi:10.1136/jmg.2009.071688

Table 1. Reports of L1CAM Mutations in Patients with L1 Syndrome and Hirschsprung Disease

Reference

Mutation

Okamoto et al., 1997

c.2421_2422de 
Vits et al., 1998

c. $1895 \mathrm{G}>\mathrm{C}$

Parisi et al., 2002

Okamoto et al., 2004 (case 1)

Okamoto et al., 2004 (case 2)

Okamoto et al., 2004 (case 3)

Basel-Vanagaite et al., 2006 (case 1)

Tegay et al., 2007

Nakakimura et al., 2008

c. $2254 \mathrm{G}>\mathrm{A}$

c. $1939+5 \mathrm{G}>\mathrm{H}$

c. $1939+5 \mathrm{G}>\mathrm{A}$

c. $2974 \mathrm{C}>\mathrm{T}$

c. $719 \mathrm{C}>\mathrm{T}$

$\mathrm{Xq} 28$ microde

c. $92 \mathrm{~T}>\mathrm{C}$

Jackson et al., 2009

c. $1672 \mathrm{C}>\mathrm{T}$

Griseri et al., 2009

c. $2265 \mathrm{delC}$

Fernandez et al., 2012

Takenouchi et al., 2012

The propositus

c. $2092 \mathrm{G}>\mathrm{A}$

c. $61 \mathrm{C}>\mathrm{T}$

c. $934 \mathrm{~T}>\mathrm{C}$

Fn, fibronectin type III domain; Ig, immunoglobulin domain. CADD score was not calculated for deletions.

Fn, fibronecti

Figure 1.

Schematic of the L1CAM protein with immunoglobulin domain (IG); fibronectin type III domain (Fn); transmembrane domain (TM); cytoplasm of cell (cyto).

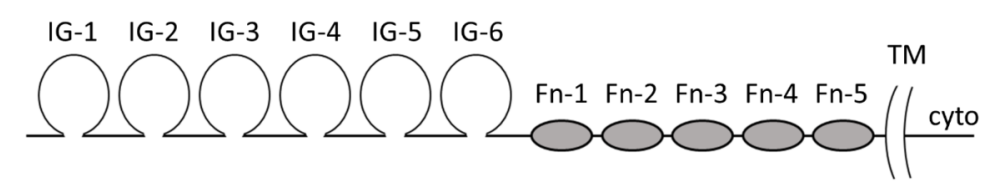

Figure 2 .

Clinical and diagnostic imaging characteristics of L1 Syndrome. (A) Magnetic resonance imaging of head with ventriculomegaly and absent corpus callosum. (B) Bilaterally adducted thumbs. (C) Abdominal radiograph with dilated loops, characteristic of HD.

A

\section{Hosted file}

image2.emf available at https://authorea.com/users/370466/articles/489114-hirschsprungdisease-in-an-infant-with-l1-syndrome-report-of-a-new-case-and-a-novel-11cam-variant

B C 


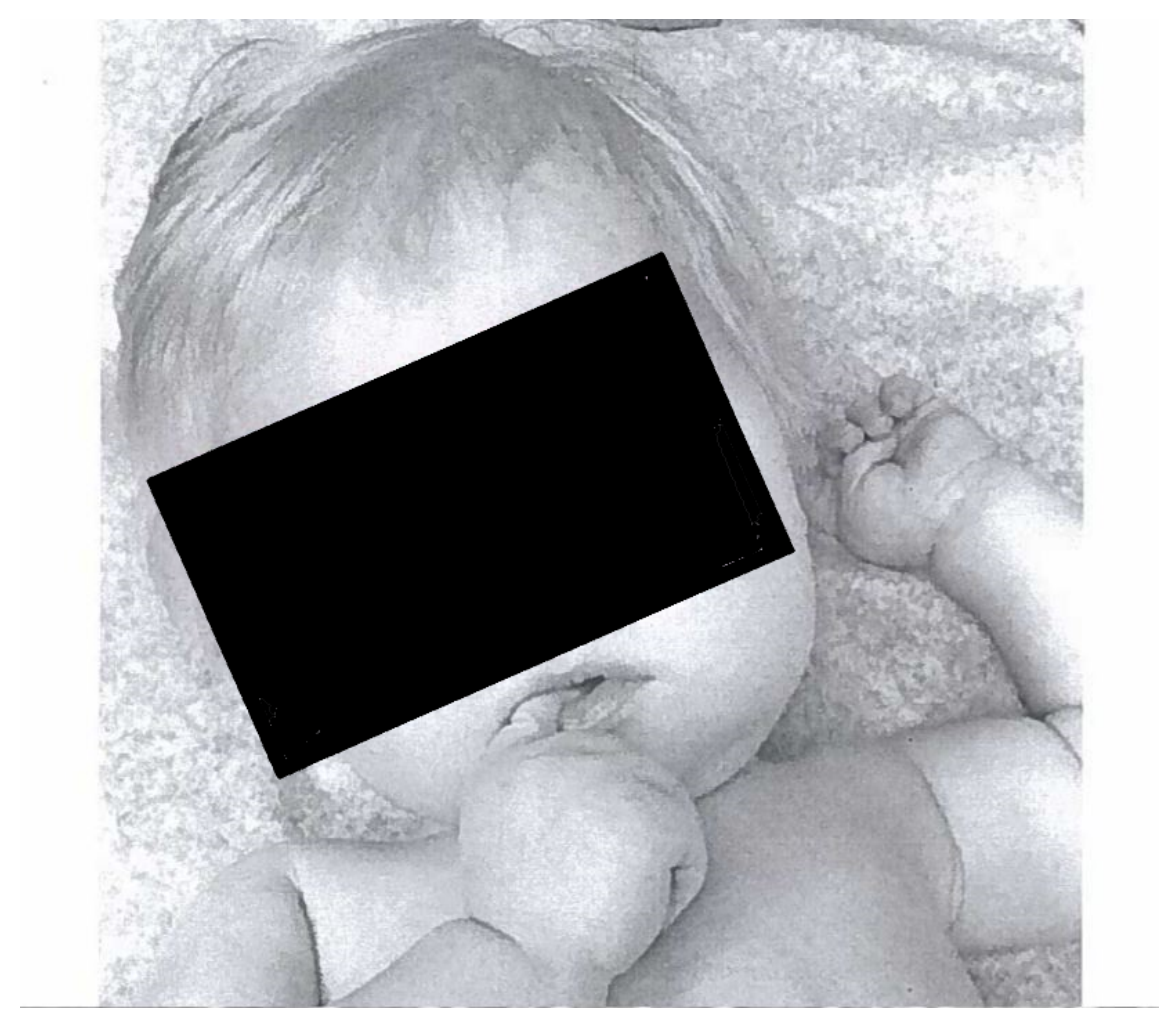




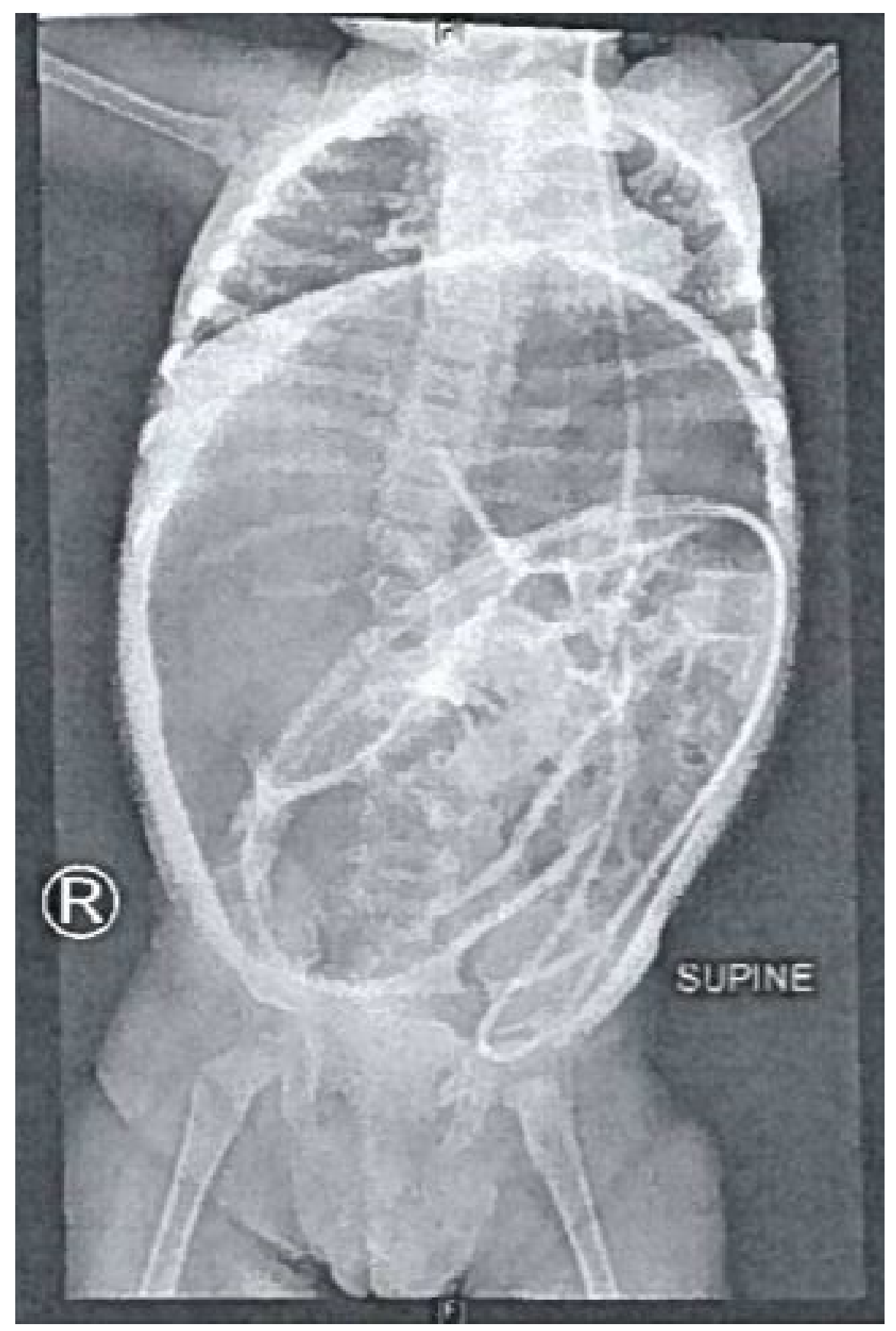

Figure 3 .

Histopathology of colon biopsy at 10x magnification. (A) Hematoxylin and eosin stain demonstrating rectal mucosa with absence of ganglion cells in the submucosal plexus and hypertrophic nerve bundles (*). (B) Calretinin immunohistochemical stain revealing absence of neurofibrils within the lamina propria or submucosal ganglion cells, a staining pattern consistent with Hirschsprung disease.

A 


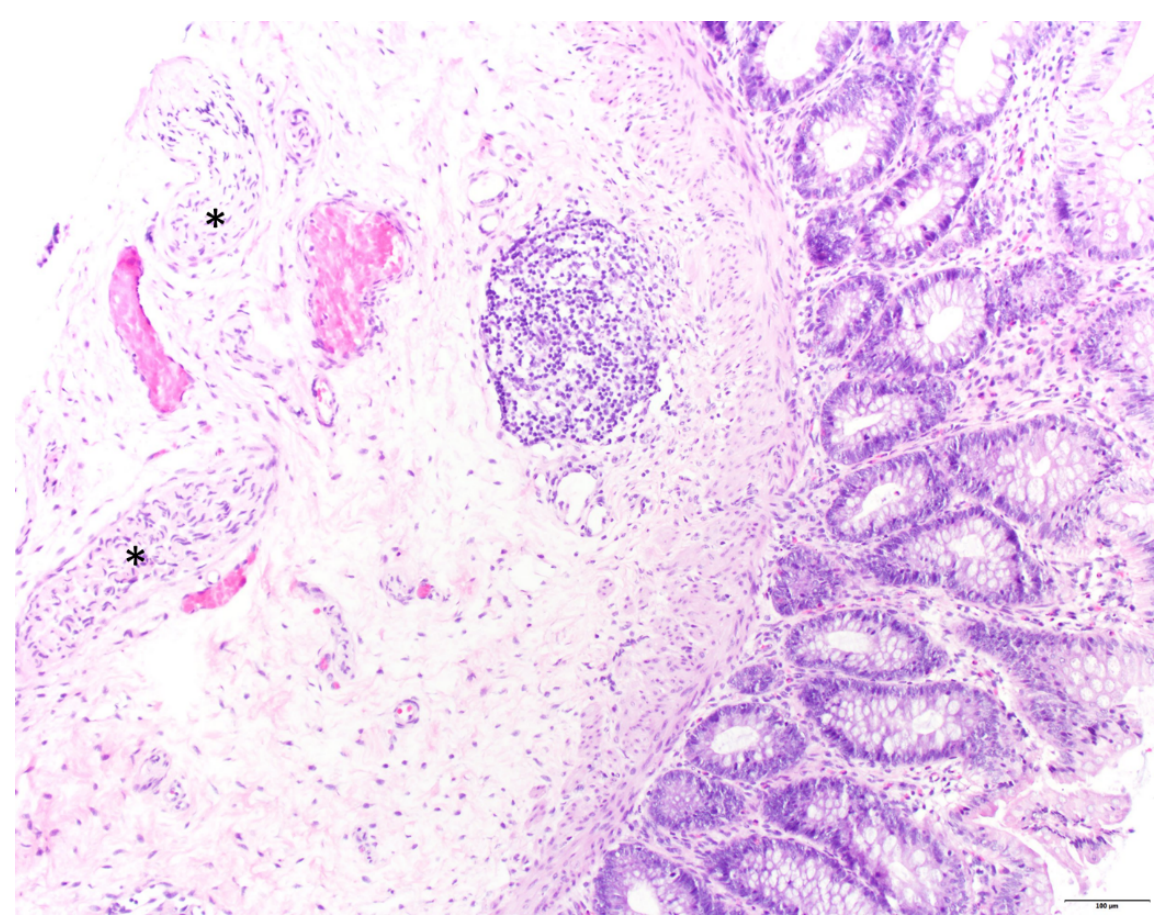

B

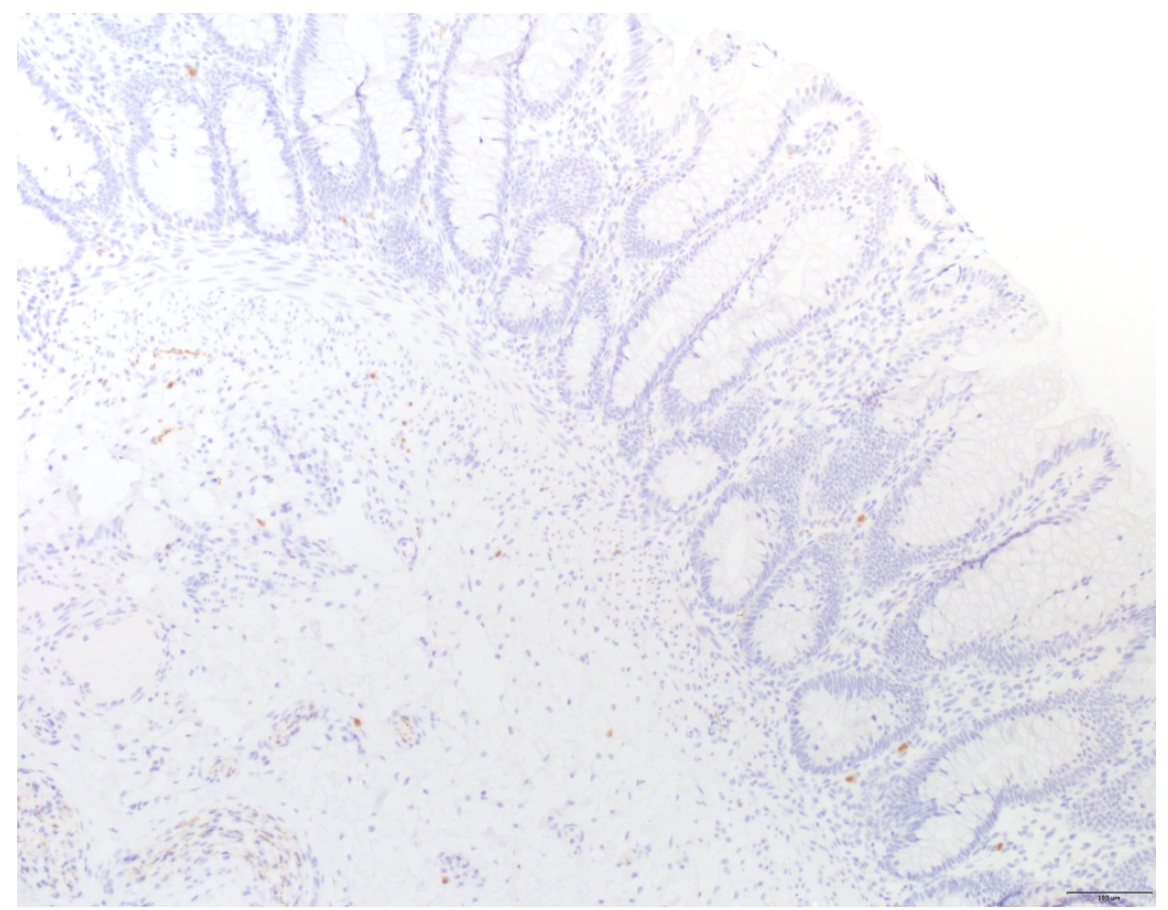

Figure 4.

Amino acid structures of cysteine and arginine. Note charged vs. uncharged side groups. 


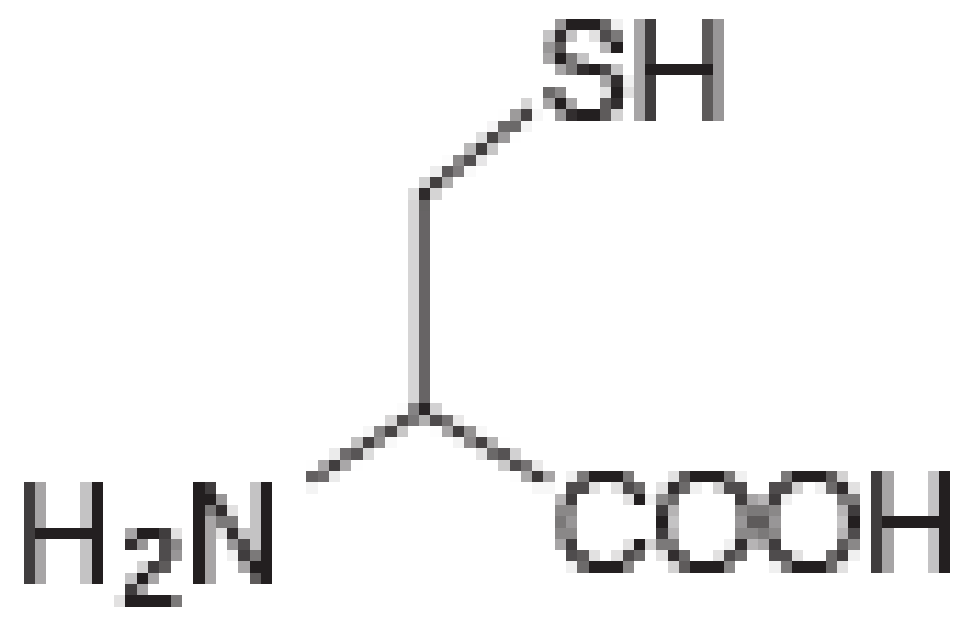

Cysteine (Cys, C) 


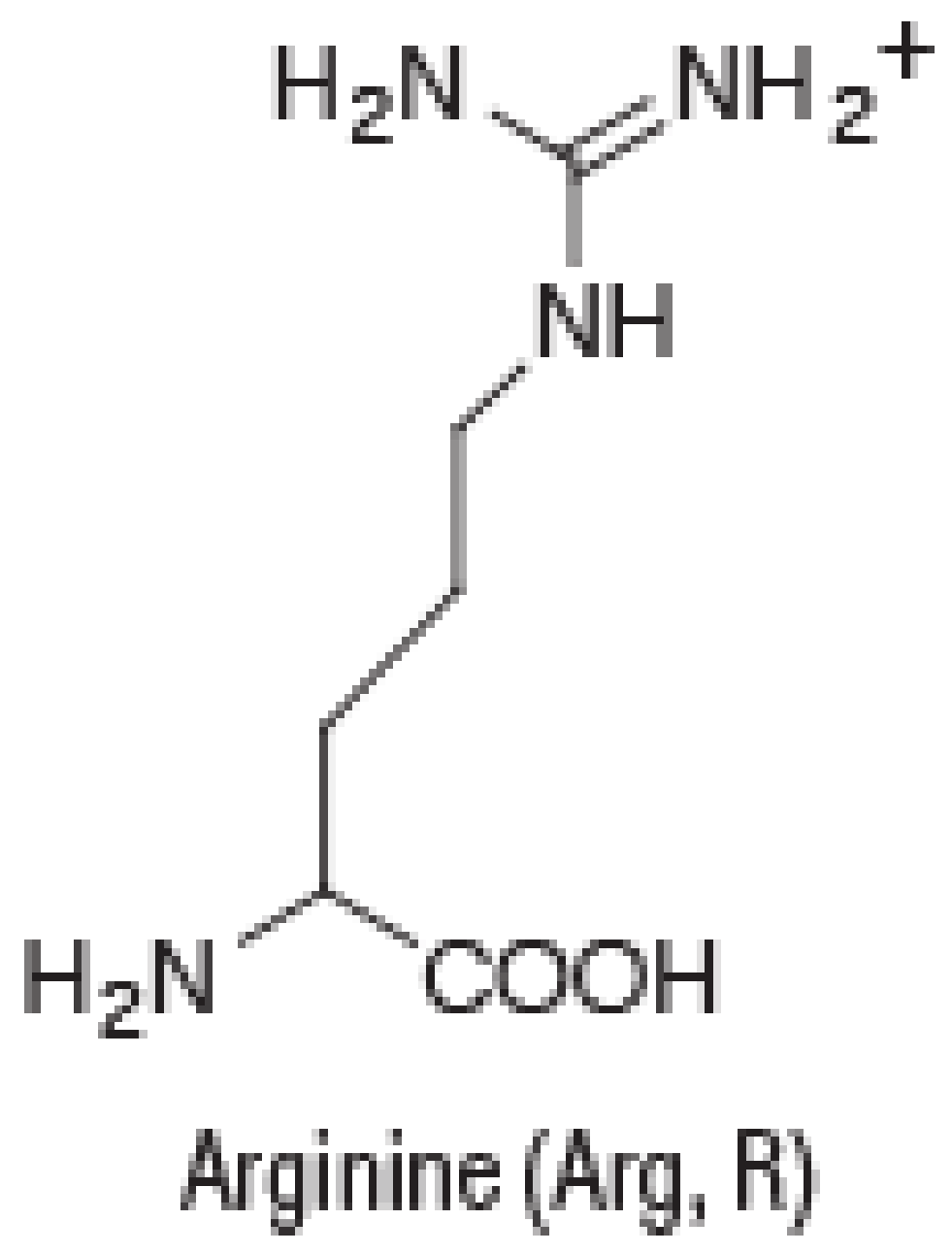

